\title{
Diego de San Pedro, La Prison d'amour (1552)
}

\author{
Maria Colombo Timelli
}

\section{(2) OpenEdition}

\section{Journals}

\section{Edizione digitale}

URL: http://journals.openedition.org/studifrancesi/9460

DOI: $10.4000 /$ studifrancesi.9460

ISSN: 2421-5856

\section{Editore}

Rosenberg \& Sellier

\section{Edizione cartacea}

Data di pubblicazione: 1 décembre 2007

Paginazione: 637-638

ISSN: 0039-2944

\section{Notizia bibliografica digitale}

Maria Colombo Timelli, «Diego de San Pedro, La Prison d'amour (1552)», Studi Francesi [Online], 153 (LI | III) | 2007, online dal 30 novembre 2015, consultato il 12 janvier 2021. URL: http://

journals.openedition.org/studifrancesi/9460 ; DOI: https://doi.org/10.4000/studifrancesi.9460

Questo documento è stato generato automaticamente il 12 janvier 2021.

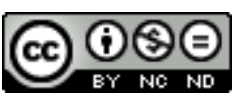

Studi Francesi è distribuita con Licenza Creative Commons Attribuzione - Non commerciale - Non opere derivate 4.0 Internazionale. 


\title{
Diego de San Pedro, La Prison d'amour (1552)
}

\author{
Maria Colombo Timelli
}

\section{NOTIZIA}

DIEGO DE SAN PEDRO, La Prison d'amour (1552), édition établie et annotée par Véronique DUCHÉ-GAVET, Paris, Champion, 2007 («Textes de la Renaissance», 119), pp. 250.

1 La Cárcel de amor, 'novela sentimental' di Diego de San Pedro pubblicata a Siviglia nel 1492, conobbe, al pari di altri romanzi spagnoli contemporanei, un successo europeo di lunga durata. Tradotta dapprima in italiano da Lelio Manfredi (1514) su richiesta di Isabella d'Este, passò poi in francese ad opera di Francois Dassy (1525), quindi in inglese grazie a John Bourchier (1533 circa, traduzione fondata sul testo di Dassy), poi di nuovo in francese con Gilles Corrozet (1552), infine - ma ben più tardi - in tedesco (1625). Le edizioni, monolingui e bilingui (spagnolo-francese) si moltiplicarono parimenti, coprendo un arco temporale che va da fine ' 400 a metà del xvII secolo. Véronique Duché-Gavet pubblica ora la traduzione di Corrozet (non si può mancare, allora, di segnalare l'ambiguità del titolo adottato: La Prison d'amour qui edita non è certamente quella di Diego de San Pedro), testo interessante senza alcun dubbio, ma testimonianza di una seconda ricezione francese: la scelta di questa redazione non è giustificata - si veda l'introduzione, p. XVIII -, anche se si spiega, con ogni probabilità, per la semplicità del lavoro critico; la traduzione di François Dassy infatti è trasmessa da nove manoscritti cui si aggiungono almeno sette stampe tra il 1525 e il 1533. D'altra parte, Corrozet non è nuovo a queste imprese editoriali: già nel 1546 aveva pubblicato l'Histoire d'Aurelio et Isabel, ancora una seconda traduzione (dopo quella di Jean Beaufilz, apparsa nel 1529 con il titolo Le Jugement d'amour) di una 'novela sentimental' spagnola di fine Quattrocento: il Grisel y Mirabela di Juan de Flores.

2 L'introduzione di Duché-Gavet è sintetica, ma fa il punto sulle questioni essenziali: colloca la Cárcel nella produzione di Diego de San Pedro e nel quadro più generale del 
romanzo sentimentale spagnolo, traccia la ricezione europea dell'opera, per fermarsi poi sulla duplice traduzione francese. Segue un rapido excursus sull'uso dell'allegoria, la 'religio amoris', tema centrale del romanzo, e l'apologia delle donne, argomento con il quale San Pedro in Spagna, ma poi Dassy e Corrozet in Francia, prendono posizione nella cosiddetta 'Querelle des Amyes'. Utile l'elenco delle 'éditions' (in realtà: manoscritti e stampe) nelle diverse lingue europee, fornito alle pp. XXIX-XXXVII. Nelle tre paginette scarse intitolate «Notes sur l'établissement du texte» (pp. XxxIX-XLI) si troveranno poche e scarne indicazioni sulla lingua della traduzione di Corrozet, nonché i principi seguiti per la presentazione del testo: alcune scelte, ad esempio riguardo alla punteggiatura («nous reproduisons la ponctuation et les majuscules de l'original, y compris les points équivalant à une simple virgule», afferma Duché-Gavet, salvo poi continuare: «en revanche, nous substituons aux deux points marquant une pause forte un point virgule...», p. XLI), appaiono francamente poco coerenti.

3 L'edizione ripropone la stampa di Corrozet pur modificandone la 'mise en page': qui il testo francese occupa la parte superiore, quello spagnolo (in corpo ridotto) la parte inferiore della pagina; i rinvii alla paginazione originale (in $8^{\circ}$ ) sono indicati soltanto per la prima metà del foglio di stampa. Le note e i commenti al testo si trovano a piè di pagina, mentre l'apparato critico è collocato alle pp. 169-198; a questo proposito si rileverà che le varianti proposte rimandano alle edizioni reperibili nelle biblioteche parigine, ai manoscritti che l'editrice ha potuto consultare (sic) «et à la première traduction de François Dassy» (nota a p. 169): salvo errore da parte mia, i due testi coincidono, in quanto i manoscritti non possono essere che quelli della traduzione di Dassy. Peraltro, la segnatura del ms. 'fr 4382', da cui è tratta una 'Lettre de Laureole au roy' in prosa (p. 186), va corretta in 'fr 24382'.

4 L'edizione comprende i complementi abituali: la bibliografia recente (posteriore a quella fornita da Keith whinNom, The Spanish Sentimental Romance, 1440-1330, Londra, 1983) alle pp. XXVII-XXVIII, l'Index nominum (pp. 199-200), l'Index rerum (pp. 201-203), l'indice degli autori citati (pp. 205-206); il glossario (pp. 207-232). Un dossier iconografico alle pp. 153-168 riunisce le incisioni delle due edizioni di Galliot Du Pré 1526 e 1527 (rispettivamente 13 e 4 'bois gravés', alcuni di reimpiego), ma va ricordato che si tratta di stampe della traduzione di Dassy, dal momento che le edizioni bilingui di Corrozet non sono illustrate. Tre 'annexes' occupano le ultime pagine del volume; si tratta della strofa liminare del ms. BnF fr. 7552 (ancora una volta, si tratta del testo di François Dassy), l'épître liminaire' in prosa dell'edizione Du Pré del 1526 (idem), e infine - abbastanza sorprendentemente, dal momento che manca qualsiasi informazione introduttiva a questi testi - l'extrait' della Prison d'amour pubblicato nella «Bibliothèque Universelle des Romans», luglio 1779, vol. I, fondato sull'edizione Du Pré 1526.

5 Un'edizione comoda, quella fornita da Véronique Duché-Gavet, ma che andrà utilizzata con prudenza, almeno fino a che non sarà disponibile anche la prima traduzione francese, quella versione più volte citata di Francois Dassy che, non va dimenticato se non si vuole falsare il quadro cronologico e quindi letterario, costituisce la vera ricezione di questa 'novela' spagnola sul suolo francese, come attesta tra l'altro la duplice diffusione, manoscritta e a stampa, certo non minore di quella del testo di Corrozet. 